\title{
含邻氟苯基的 1,2,4-三唑类衍生物的合成及杀菌活性研究
}

\author{
童建预 ${ }^{a}$ 石延霞 ${ }^{c} \quad$ 刘幸海*,b 孙娜波 ${ }^{a, b}$ 李宝聚*,c \\ ( ${ }^{a}$ 浙江树人大学生物与环境学院 杭州 310015) \\ ( ${ }^{b}$ 浙江工业大学化学工程与材料学院 杭州 310014) \\ $\left({ }^{c}\right.$ 中国农业科学院蔬菜花卉研究所 北京 100014)
}

\begin{abstract}
摘要 以邻氟苯甲酸、水合肼、苯基异硫氧酸酯等为原料, 通过多步反应制备了一系列邻氟苯基联三唑类衍生物, 并 考察了微波辐射对反应的影响. 产物结构均经过 ${ }^{1} \mathrm{H}$ NMR, MS 和元素分析确证; 对所有化合物进行了活体杀菌活性测 试, 结果表明大部分化合物对黄瓜褐斑病、黄瓜核菌病、黄瓜霜霓病、黄瓜细菌性角斑病均具有较好的防效. 关键词 1,2,4-三唑; 合成; 杀菌活性; 邻氟苯甲酸
\end{abstract}

\section{Synthesis and Fungicidal Activity of 1,2,4-Triazole Derivatives Containing 2-Fluorophenyl Moiety}

\author{
Tong, Jianying ${ }^{a} \quad$ Shi, Yanxia $^{c} \quad$ Liu, Xinghai*, $^{*} \quad$ Sun, Nabo ${ }^{a, b} \quad$ Li, Baoju ${ }^{*, c}$ \\ ( ${ }^{a}$ College of Biology and Environmental Engineering, Zhejiang Shuren University, Hangzhou 310015) \\ $\left({ }^{b}\right.$ College of Chemical Engineering and Materials Science, Zhejiang University of Technology, Hangzhou 310014) \\ ( ${ }^{c}$ Institute of Vegetables and Flowers, Chinese Academy of Agricultural Sciences, Beijing 100014)
}

\begin{abstract}
A series of novel 1,2,4-triazole derivatives were synthesized from $o$-fluorobenzoic acid, hydrazine hydrate, phenyl isothiocyanate by multi-step reactions. Their structures were characterized by ${ }^{1} \mathrm{H}$ NMR, MS and elemental analyses. The target compounds were evaluated for their fungicidal activities against Corynespora cassiicola, Pseudomonas syringae pv. Lachrymans, Pseudoperonospora cubensis and Sclerotinia sclerotiorum in vivo, and the results indicated that some of the title compounds displayed excellent fungicidal activities.
\end{abstract}

Keywords 1,2,4-triazole; synthesis; fungicidal activity; $o$-fluorobenzoic acid

近年来，由于现代农业和环境保护对农药的要求日 益提高, 已经由传统的杀死转变成为生物调控. 杂环化 合物由于其多变的结构和广泛的生物活性使得在农药 的开发中受到广泛的关注, 尤其是含氮杂环. 1,2,4-三唑 类化合物因具有除草 ${ }^{[1]}$ 、杀菌 ${ }^{[2]}$ 、杀虫 ${ }^{[3]}$ 、抗癌 ${ }^{[4]}$ 、抗 炎 ${ }^{[5]}$ 、抗菌 ${ }^{[6]}$ 、抗惊厥 ${ }^{[7]}$ 等多样性的生物活性而受到广泛 关注. 一些含 $1,2,4$-三唑环的化合物已经开发成功许多 商品化的农药和医药(图 1), 如除草剂氟胺草唑 (flupoxam)、杀菌剂氟硅唑(Flusilazole)、抗菌消炎药物 氟康唑(Fluconazole)、抗癌药物阿那曲唑(Anastrozole) 等. 氟原子在生物体内具有模拟效应、电子效应、阻碍
效应、渗透效应等, 在化合物中引入氟原子有可能产生 优异的生物活性，因而在医药和农药创制研究中屡见报 道 ${ }^{[8][9]}$.

为了进一步从 1,2,4-三唑类化合物中寻找高活性的 先导化合物, 本文采用活性亚结构拼接的方法, 设计了 一系列新型的 1,2,4-三唑类衍生物; 以邻氟苯甲酸为原 料引入氟原子, 经酯化、肼解、缩合、环化和烷基化等 多步反应合成了目标产物; 经核磁、质谱及元素分析等 表征手段确证了其结构, 并对其进行了活体杀菌活性的 评价，结果表明部分化合物对测试菌种具有优异的防 效. 目标化合物的合成路线如 Scheme 1 所示.

\footnotetext{
*E-mail:xhliu@zjut.edu.cn

Received July 16, 2012; revised August 10, 2012; published online August 15, 2012.

Project supported by the National Natural Science Foundation of China (No. 21002090), the Key Innovation Team of Science and Technology in Zhejiang Province (No. 2010R50018-06), and the National Key Technologies R\&D Program (No. 2011BAE06B03-01).

国家自然科学基金(No. 21002090)、浙江省重点创新团队(No. 2010R50018-06)、“十二五” 国家科技支撑计划(No. 2011BAE06B03-01)资助项目，
} 
<smiles>OC(c1ccc(F)cc1)n1cncn1</smiles>

氟康唑(Fluconazole)<smiles>Fc1ccc([Si](Cn2cncn2)(c2ccc(F)cc2)c2ccc(F)cc2)cc1</smiles>

氟硅莝(Flusilazole)

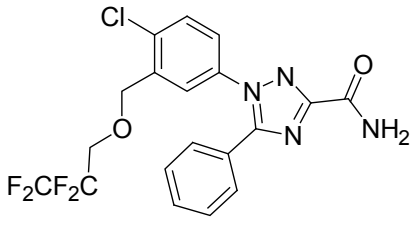

氟胺草唑(Flupoxam)

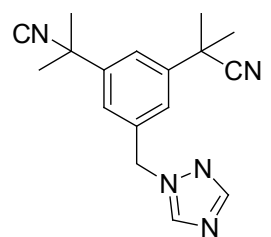

阿那曲唑(Anastrozole)
图 1 一些商品化的三唑类医药和农药

Figure 1 Some commercial medicines and pesticides about triazole

\section{1 实验部分}

\section{1 试剂与仪器}

试剂均采用分析纯试剂. 未经处理, 直接使用.

北京泰克仪器有限公司 X-4 数字显示显微熔点仪, 温度计未校正; BRUKER AV-400 型核磁共振仪 (400 $\mathrm{MHz})\left(\mathrm{CDCl}_{3}\right.$ 为溶剂, TMS 为内标); Thermo Finnigan LCQ Advantage 型质谱仪; Yanaco MT-3CHN 元素分析 仪; 美国 CEM 公司 Discovery ${ }^{\mathrm{TM}}$ 微波合成仪.

\section{2 化合物的合成}

邻氟苯甲酸甲酯和邻氟苯甲酰肼参考文献[10]方法 由邻氟苯甲酸、氯化亚砜、水合胇等合成, 苯基异硫氰 酸酯参考文献[11]制备.

1.2.15-(2-氟苯基)-4-苯基-4H-1,2,4-三唑-3-硫醇 (4) 的合成

$100 \mathrm{~mL}$ 三口瓶中加入 $10 \mathrm{mmol}$ 邻氟苯甲酰肼和 40 $\mathrm{mL}$ 无水乙醇, 滴入 $10 \mathrm{mmol}$ 苯基异硫氰酸酯, 回流 3 $h$, 出现大量白色固体. 冷却过滤, 烘干后直接用于下一
步反应. $50 \mathrm{~mL}$ 圆底烧瓶中加入 $10 \mathrm{mmol}$ 化合物 3 和 15 $\mathrm{mL} 2 \mathrm{~mol} / \mathrm{L} \mathrm{NaOH}$ 溶液, 回流 $4 \mathrm{~h}, 4 \mathrm{~mol} / \mathrm{L}$ 盐酸中和至中 性，产生大量白色固体 $\mathbf{4}$, 过滤，干燥，用乙醇和水重结 晶的白色针状晶体, 产率 $73.5 \%$. m.p. $111 \sim 112{ }^{\circ} \mathrm{C} ;{ }^{1} \mathrm{H}$ NMR $\left(\mathrm{CDCl}_{3}, 400 \mathrm{MHz}\right) \delta: 6.99$ (t, $\left.J=9.4 \mathrm{~Hz}, 1 \mathrm{H}, \mathrm{ArH}\right)$, $7.18(\mathrm{t}, J=7.6 \mathrm{~Hz}, 1 \mathrm{H}, \mathrm{ArH}), 7.27 \sim 7.29(\mathrm{~m}, 2 \mathrm{H}, \mathrm{ArH})$, $7.38 \sim 7.46$ (m, 5H, ArH), 11.94 (brs, 1H, SH); MS (ESI) $m / z: 270(\mathrm{M}-1)^{-}$. Anal. calcd for C 61.98, H 3.72, N 15.49; found C 62.14, H 3.43, N 15.74.

1.2.2 3-(取代苹硫基)-5-(2-氟苯基)-4-苯基-4H-1,2,4三唑 $(5 \mathbf{a} \sim 5 \mathbf{j})$ 的合成通法

$25 \mathrm{~mL}$ 圆底烧瓶中加入 $2 \mathrm{mmol}$ 化合物 $\mathbf{4}, 2 \mathrm{mmol}$ 取 代氯芐, $8 \mathrm{~mL}$ DMF 和 $2.4 \mathrm{mmol}$ 的 $\mathrm{K}_{2} \mathrm{CO}_{3}$, 室温搅拌, $\mathrm{TLC}$ 检测反应进度. 反应完毕后, 反应液倾入 $30 \mathrm{~mL}$ 冰 水中, 析出固体, 过滤, 干燥, 乙醇重结晶的产物.

3-(2,4-二氯芳硫基)-5-(2-氟苯基)-4-苯基-4H-1,2,4三唑(5a): 白色晶体, 产率 91\%. m.p. $115 \sim 116{ }^{\circ} \mathrm{C} ;{ }^{1} \mathrm{H}$ NMR $\left(\mathrm{CDCl}_{3}, 400 \mathrm{MHz}\right) \delta$ : $4.57\left(\mathrm{~s}, 2 \mathrm{H}, \mathrm{SCH}_{2}\right), 6.94(\mathrm{t}$, $J=9.2 \mathrm{~Hz}, 1 \mathrm{H}, \mathrm{ArH}), 7.03(\mathrm{t}, J=7.3 \mathrm{~Hz}, 2 \mathrm{H}, \mathrm{ArH}), 7.17 \sim$ 7.20 (m, 2H, ArH), $7.34 \sim 7.41$ (m, 5H, ArH), $7.53 \sim 7.59$ (m, 2H, ArH); MS (ESI) $m / z: 431(\mathrm{M}+1)^{+}$. Anal. calcd for C 58.61, H 3.28, N 9.76; found C 58.98, H 3.12, N 10.09.

3-(3-氯芐硫基)-5-(2-氟苯基)-4-苯基-4H-1,2,4-三唑 (5b): 白色晶体，产率 83\%. m.p. 53 54 ${ }^{\circ} \mathrm{C} ;{ }^{1} \mathrm{H}$ NMR $\left(\mathrm{CDCl}_{3}, 400 \mathrm{MHz}\right) \delta: 4.46\left(\mathrm{~s}, 2 \mathrm{H}, \mathrm{SCH}_{2}\right), 6.93$ (t, $J=9.5$ $\mathrm{Hz}, 1 \mathrm{H}, \mathrm{ArH}), 7.02(\mathrm{t}, J=7.6 \mathrm{~Hz}, 2 \mathrm{H}, \mathrm{ArH}), 7.17 \sim 7.24$ (m, 3H, ArH), $7.33 \sim 7.42(\mathrm{~m}, 6 \mathrm{H}, \operatorname{ArH}), 7.57(\mathrm{t}, J=$ $5.9 \mathrm{~Hz}, 1 \mathrm{H}, \mathrm{ArH})$; MS (ESI) $m / z: 397(\mathrm{M}+1)^{+}$. Anal. calcd for $\mathrm{C} 63.71, \mathrm{H} 3.82, \mathrm{~N} 10.61$; found $\mathrm{C} 63.45, \mathrm{H} 3.52, \mathrm{~N}$ 10.43 .

3-(3-氰基苄硫基)-5-(2-氟苯基)-4-苯基-4H-1,2,4-三 唑(5c)：白色晶体，产率 86\%. m.p. 95 96 ${ }^{\circ} \mathrm{C} ;{ }^{1} \mathrm{H}$ NMR $\left(\mathrm{CDCl}_{3}, 400 \mathrm{MHz}\right) \delta: 4.49$ (s, 2H, $\left.\mathrm{SCH}_{2}\right), 6.92$ (t, $J=8.8$ $\mathrm{Hz}, 1 \mathrm{H}, \mathrm{ArH}), 7.03$ (t, $J=6.9 \mathrm{~Hz}, 2 \mathrm{H}, \mathrm{ArH}), 7.17$ (t, $J=$

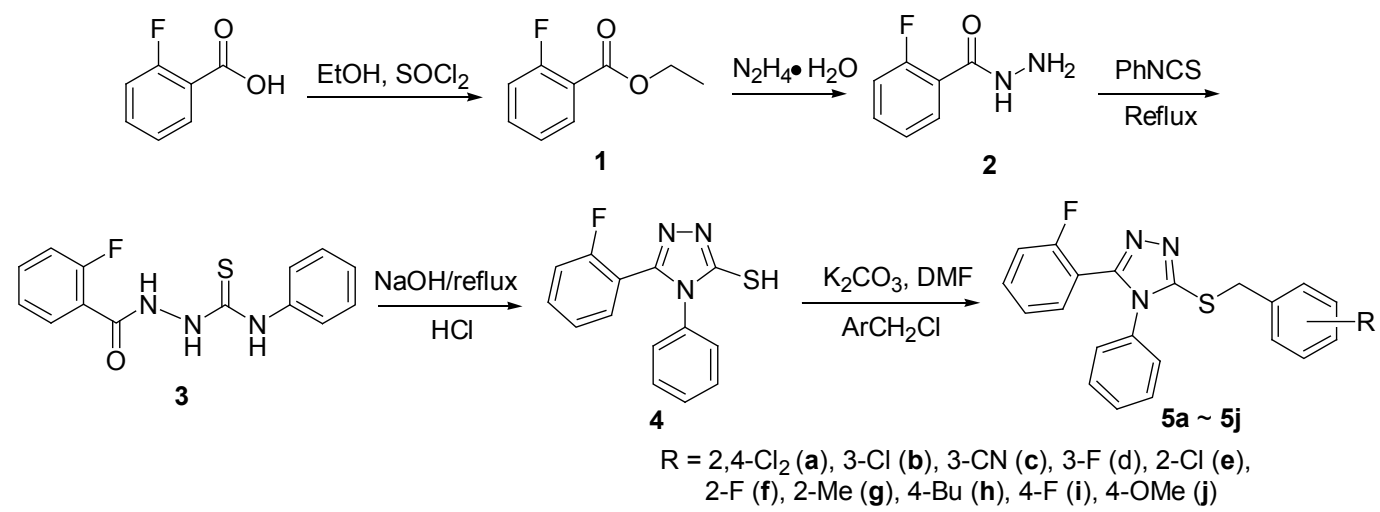

Scheme 1 
$7.6 \mathrm{~Hz}, 1 \mathrm{H}, \mathrm{ArH}), 7.35 \sim 7.41(\mathrm{~m}, 5 \mathrm{H}, \mathrm{ArH}), 7.53 \sim 7.57$ (m, 2H, ArH), $7.65 \sim 7.66$ (m, 2H, ArH); MS (ESI) $m / z$ : $387(\mathrm{M}+1)^{+}$. Anal. calcd for C 68.38, H 3.91, N 14.50; found $\mathrm{C} 68.55, \mathrm{H} 4.03, \mathrm{~N} 14.41$.

3-(3-氟芐硫基)-5-(2-氟苯基)-4-苯基-4H-1,2,4-三唑 (5d): 白色晶体, 产率 $89 \%$. m.p. $87 \sim 88{ }^{\circ} \mathrm{C} ;{ }^{1} \mathrm{H}$ NMR $\left(\mathrm{CDCl}_{3}, 400 \mathrm{MHz}\right) \delta: 4.49$ (s, 2H, $\left.\mathrm{SCH}_{2}\right), 6.94$ (q, $J=8.6$ $\mathrm{Hz}, 2 \mathrm{H}, \mathrm{ArH}), 7.03 \sim 7.08(\mathrm{~m}, 3 \mathrm{H}, \mathrm{ArH}), 7.13 \sim 7.25(\mathrm{~m}$, $3 \mathrm{H}, \mathrm{ArH}), 7.34 \sim 7.40(\mathrm{~m}, 4 \mathrm{H}, \mathrm{ArH}), 7.58(\mathrm{t}, J=7.4 \mathrm{~Hz}$, 1H, ArH), $7.65 \sim 7.66$ (m, 2H, ArH); MS (ESI) $m / z: 380$ $(\mathrm{M}+1)^{+}$. Anal. calcd for C 66.48, H 3.98, N 11.07; found C 66.76, H 4.03, N 10.89.

3-(2-氯芐硫基)-5-(2-氟苯基)-4-苯基-4H-1,2,4-三唑 (5e): 白色晶体, 产率 $92 \%$. m.p. $113 \sim 114{ }^{\circ} \mathrm{C} ;{ }^{1} \mathrm{H}$ NMR $\left(\mathrm{CDCl}_{3}, 400 \mathrm{MHz}\right) \delta: 4.62\left(\mathrm{~s}, 2 \mathrm{H}, \mathrm{SCH}_{2}\right), 6.93$ (t, $J=8.5$ $\mathrm{Hz}, 1 \mathrm{H}, \mathrm{ArH}), 7.01$ (d, J=7.6 Hz, 2H, ArH), 7.16 7.24 (m, 3H, ArH), $7.31 \sim 7.40(\mathrm{~m}, 5 \mathrm{H}, \mathrm{ArH}), 7.53 \sim 7.57(\mathrm{~m}$, $2 \mathrm{H}, \mathrm{ArH})$; MS (ESI) $m / z: 397(\mathrm{M}+1)^{+}$. Anal. calcd for $\mathrm{C}$ 63.71, H 3.82, N 10.61; found C 64.01, H 4.13, N 10.54.

3-(2-氟芐硫基)-5-(2-氟苯基)-4-苯基-4H-1,2,4-三唑 (5f): 白色晶体, 产率 74\%. m.p. 111 112 ${ }^{\circ} \mathrm{C} ;{ }^{1} \mathrm{H}$ NMR $\left(\mathrm{CDCl}_{3}, 400 \mathrm{MHz}\right) \delta: 4.54$ (s, 2H, $\left.\mathrm{SCH}_{2}\right), 6.93$ (t, $J=9.0$ $\mathrm{Hz}, 1 \mathrm{H}, \mathrm{ArH}), 6.99 \sim 7.09$ (m, 4H, ArH), 7.18 (t, $J=7.6$ $\mathrm{Hz}, 1 \mathrm{H}, \mathrm{ArH}), 7.22 \sim 7.28(\mathrm{~m}, 1 \mathrm{H}, \mathrm{ArH}), 7.32 \sim 7.40(\mathrm{~m}$, 4H, ArH), $7.48(\mathrm{t}, J=6.1 \mathrm{~Hz}, 1 \mathrm{H}, \mathrm{ArH}), 7.48$ (t, $J=5.8$ $\mathrm{Hz}, 1 \mathrm{H}, \mathrm{ArH})$; MS (ESI) $m / z: 380(\mathrm{M}+1)^{+}$. Anal. calcd for C 66.48, H 3.98, N 10.01; found C 66.75, H 4.13, N 9.97.

3-(2-甲基苄硫基)-5-(2-氟苯基)-4-苯基-4H-1,2,4-三 唑(5g): 白色晶体, 产率 93\%. m.p. 90 91 ${ }^{\circ} \mathrm{C} ;{ }^{1} \mathrm{H}$ NMR $\left(\mathrm{CDCl}_{3}, 400 \mathrm{MHz}\right) \delta: 2.33$ (s, 3H, Me), $4.52\left(\mathrm{~s}, 2 \mathrm{H}, \mathrm{SCH}_{2}\right)$, $6.93(\mathrm{t}, J=9.2 \mathrm{~Hz}, 1 \mathrm{H}, \operatorname{ArH}), 6.99$ (d, $J=7.2 \mathrm{~Hz}, 2 \mathrm{H}$, ArH), $7.11 \sim 7.20(\mathrm{~m}, 4 \mathrm{H}, \mathrm{ArH}), 7.28 \sim 7.39(\mathrm{~m}, 5 \mathrm{H}, \mathrm{ArH})$, $7.56(\mathrm{t}, J=5.5 \mathrm{~Hz}, 1 \mathrm{H}, \mathrm{ArH})$; MS (ESI) $m / z: 376(\mathrm{M}+1)^{+}$. Anal. calcd for C 70.38, H 4.83, N 11.19; found C 70.54, H 4.66, N 10.97.

3-(4-叔丁基芐硫基)-5-(2-氟苯基)-4-苯基-4H-1,2,4三唑(5h): 白色晶体, 产率 $89 \%$. m.p. $183 \sim 184{ }^{\circ} \mathrm{C} ;{ }^{1} \mathrm{H}$ $\mathrm{NMR}\left(\mathrm{CDCl}_{3}, 400 \mathrm{MHz}\right) \delta: 1.29$ (s, 9H, Me), 4.49 (s, 2H, $\left.\mathrm{SCH}_{2}\right), 6.95$ (t, $\left.J=9.4 \mathrm{~Hz}, 1 \mathrm{H}, \mathrm{ArH}\right), 6.99(\mathrm{~d}, J=7.5 \mathrm{~Hz}$, 2H, ArH), $7.18(\mathrm{t}, J=7.6 \mathrm{~Hz}, 1 \mathrm{H}, \mathrm{ArH}), 7.25 \sim 7.28(\mathrm{~m}$, $3 \mathrm{H}, \mathrm{ArH}), 7.35 \sim 7.40(\mathrm{~m}, 5 \mathrm{H}, \mathrm{ArH}), 7.56(\mathrm{t}, J=5.7 \mathrm{~Hz}$, $1 \mathrm{H}, \mathrm{ArH}) ; \mathrm{MS}(\mathrm{ESI}) \mathrm{m} / z$ : $418(\mathrm{M}+1)^{+}$. Anal. calcd for $\mathrm{C}$ 71.91, H 5.79, N 10.06; found C 72.08, H 5.66, N 10.34.

3-(4-氟芐硫基)-5-(2-氟苯基)-4-苯基-4H-1,2,4-三唑 (5i): 白色晶体，产率 91\%. m.p. $118 \sim 119{ }^{\circ} \mathrm{C} ;{ }^{1} \mathrm{H}$ NMR $\left(\mathrm{CDCl}_{3}, 400 \mathrm{MHz}\right) \delta: 4.48\left(\mathrm{~s}, 2 \mathrm{H}, \mathrm{SCH}_{2}\right), 6.91 \sim 6.99(\mathrm{~m}$, $3 \mathrm{H}, \operatorname{ArH}), 7.03$ (d, $J=7.8 \mathrm{~Hz}, 2 \mathrm{H}, \operatorname{ArH}), 7.18$ (t, $J=7.6$ $\mathrm{Hz}, 1 \mathrm{H}, \mathrm{ArH}), 7.32 \sim 7.41(\mathrm{~m}, 6 \mathrm{H}, \mathrm{ArH}), 7.56(\mathrm{t}, J=7.4$ $\mathrm{Hz}, 1 \mathrm{H}, \mathrm{ArH})$; MS (ESI) $m / z: 380(\mathrm{M}+1)^{+}$. Anal. calcd for C 66.48, H 3.98, N 11.07; found C 66.65, H 4.13, N 11.34 .

3-(4-甲氧基芐硫基)-5-(2-氟苯基)-4-苯基-4H-1,2,4三唑(5j): 白色晶体, 产率 $92 \%$. m.p. $123 \sim 124{ }^{\circ} \mathrm{C} ;{ }^{1} \mathrm{H}$ NMR ( $\left.\mathrm{CDCl}_{3}, 400 \mathrm{MHz}\right) \delta: 3.78$ (s, 3H, OMe), 4.48 (s, $\left.2 \mathrm{H}, \mathrm{SCH}_{2}\right), 6.82(\mathrm{~d}, J=8.5 \mathrm{~Hz}, 2 \mathrm{H}, \mathrm{ArH}), 6.93$ (t, $J=8.8$ $\mathrm{Hz}, 1 \mathrm{H}, \mathrm{ArH}), 7.04$ (d, J=7.0 Hz, 2H, ArH), 7.14 7.25 (m, 2H, ArH), $7.31 \sim 7.40$ (m, 4H, ArH), 7.56 (t, $J=7.4$ $\mathrm{Hz}, 2 \mathrm{H}, \mathrm{ArH})$; MS (ESI) $m / z: 392(\mathrm{M}+1)^{+}$. Anal. calcd for C 67.50, H 4.63, N 10.73; found C 67.81, H 4.43, N 11.01 .

\section{3 生物活性测试}

\subsection{1 材料}

黄瓜品种: 新泰密刺播在装有基质(草炭：蛭石为 $2: 1)$ 的营养钵内, 营养针规格 $10 \mathrm{~cm} \times 10 \mathrm{~cm}$, 每钭播 3 粒催芽种子, 保证每钭 2 株苗, 当苗子长至 2 叶 1 心时 待用.

接种体. 黄瓜霜霉病菌、黄瓜褐斑病菌、黄瓜细菌 性角斑病菌和黄瓜核菌病菌采自中国农业科学院蔬菜 花卉研究所保存, 具有较强的致病力.

\subsection{2 试验方法}

试验设在中国农业科学院蔬菜花卉研究所蔬菜病 理研究室试验玻璃温室内, 试验条件良好.

试验药剂. 所有测试样品均采用 $500 \mu \mathrm{g} / \mathrm{mL}$.

施药及接种方法: 黄瓜褐斑病菌、霜霉病菌采用孢 子悬浮液喷雾接种, 黄瓜细菌性角斑病菌采用菌悬液喷 雾接种, 黄瓜菌核病菌采用菌丝悬液喷雾接种. 于晴天 上午将供试药剂及对照药剂均匀喷施于黄瓜子叶上, $2 \mathrm{~h}$ 后接种病原菌, 保湿培养.

调查方法. 待对照充分发病后调查防效. 公式如下:

$$
\text { 防治效果 }(\%)=\frac{\text { 对照病情一处理病情 }}{\text { 对照病情 }} \times 100 \%
$$

\section{2 结果与讨论}

\section{1 合成}

化合物的合成以邻氟苯甲酸与氯化亚砜反应开始, 首先制得邻氟苯甲酸乙酯, 后者与水合肼一起回流生成 邻氟苯甲酰肼 2. 中间体 2 与苯基异硫氧酸酯在加热条 件下形成 $N$-苯基- $N^{\prime}$-邻氟苯基-2-基甲酰基硫艮 3; 中间 
体 3 在强碱条件下脱水环合转化为 1,2,4-三唑中间体 4 . 合成中间体 4 时, 酸化的时候要注意酸性的强度, 调节 $\mathrm{pH}=7$ 左右，否则中间体 4 的产率降低.

近些年来，微波化学由于其反应时间短、收率高、 操作方便等优点, 越来越受到化学工作者的重视 ${ }^{[12]}$. 本 文在合成目标化合物 $\mathbf{5}$ 时, 将中间体 $\mathbf{4}$ 与对叔丁基氯茮 溶于 DMF 和水的混合溶液, 以碳酸钾作为缚酸剂, 然 后置于微波辐射中间歇反应 $10 \mathrm{~min}$, 得到目标化合物, 产率达到 $90 \%$ ，与常规室温搅拌法产率(反应时间为 24 $h$ ，产率 $89 \%$ )相当，但是反应时间大大缩短.

\section{2 杀菌活性}

黄瓜褐斑病(Corynespora cassiicola)、黄瓜细菌性角 斑病(Pseudomonas syringae pv. Lachrymans)、黄瓜霜霉 病 (Pseudoperonospora cubensis) 和黄瓜菌核病 (Sclerotinia sclerotiorum)的活体杀菌活性列于表 1, 测试 浓度为 $500 \mu \mathrm{g} / \mathrm{mL}$. 由表 1 可知, 部分化合物在 500 $\mu \mathrm{g} / \mathrm{mL}$ 的剂量下对黄瓜褐斑病(Corynespora cassiicola)、 黄瓜细菌性角斑病(Pseudomonas syringae pv. Lachrymans)、黄瓜霜霉病(Pseudoperonospora cubensis) 和黄瓜 菌核病(Sclerotinia sclerotiorum)表现出较好的杀菌活性. 从表 1 中可知, 化合物 $\mathbf{5 b}(83 \%)$ 和 $5 \mathbf{d}(71 \%)$ 对黄瓜褐斑 病具有较好的活性, 与对照药异菌脲(54\%), 井冈霉素
(78\%)，甲基托布津(79\%)相当，化合物 $\mathbf{5 c}, \mathbf{5 e}, \mathbf{5 f}, \mathbf{5 i}$ 对黄 瓜褐斑病的防效都在 $60 \%$ 以上. 化合物 $5 \mathbf{b}, \mathbf{5 c}, 5 \mathrm{~d}, 5 \mathbf{i}$ 对 黄瓜细菌性角斑病的防效分别为 $72 \%, 60 \%, 68 \%, 67 \%$, 比对照药防效异菌脲 $(38 \%)$, 井冈霉素( $-11 \%)$, 甲基托 布津 $(54 \%)$ 高. 对于黄瓜霜霉病，化合物 5d (64\%)和 5 e $(66 \%)$ 的防效与对照药异菌脲 $(56 \%)$, 井冈霉素 $(56 \%)$, 甲基托布津 $(69 \%)$ 相当. 而对于黄瓜核菌病，所有化合 物的防效都没有对照药异菌脲(98\%), 井冈霉素(27\%), 甲基托布津 $(83 \%)$ 好. 从初步的构效关系分析发现：对 于黄瓜褐斑病和黄瓜细菌性角斑病, 苯环上 3-位取代活 性优于其他位置取代; 从总体上看，苯环上卤素取代基 时，活性优于烷基和烷氧基，这对进一步的设计工作具 有一定的指导意义.

\section{3 结论}

本文通过多步反应合成了一系列含氟苯基的 1,2,4三唑衍生物, 实验中发现利用微波合成法能够大大缩短 反应时间. 活体杀菌活性实验表明，部分目标化合物对 黄瓜褐斑病、黄瓜细菌性角斑病、黄瓜霜霉病和黄瓜菌 核病有较好的防效，表明设计具有一定的合理性. 尤其 是化合物 $\mathbf{5 b}$ 和 $\mathbf{5 d}$, 可作为先导化合物进行进一步优化 研究.

表 1 目标化合物的活体杀菌活性 $(500 \mathrm{mg} / \mathrm{mL})$

Table 1 Fungicidal activity of title compounds in vivo $(500 \mathrm{mg} / \mathrm{mL})$

\begin{tabular}{|c|c|c|c|c|}
\hline Compd. & Corynespora cassiicola & Pseudomonas syringae pv. Lachrymans & $\begin{array}{c}\text { Pseudoperonospora } \\
\text { cubensis }\end{array}$ & $\begin{array}{l}\text { Sclerotinia } \\
\text { sclerotiorum }\end{array}$ \\
\hline $5 \mathbf{5 a}$ & 53 & 57 & 59 & 73 \\
\hline $5 \mathbf{b}$ & 83 & 72 & 50 & 29 \\
\hline $5 c$ & 67 & 60 & 60 & 37 \\
\hline 5d & 71 & 68 & 64 & 71 \\
\hline $5 e$ & 65 & 34 & 57 & -10 \\
\hline $5 f$ & 67 & 34 & 30 & 55 \\
\hline $5 g$ & 50 & 49 & 66 & 47 \\
\hline $5 \mathrm{~h}$ & 43 & 49 & 41 & 7 \\
\hline $5 \mathbf{i}$ & 62 & 67 & 47 & 64 \\
\hline $5 \mathbf{j}$ & 14 & 4 & 43 & 13 \\
\hline 异菌脲 & 54 & 38 & 56 & 98 \\
\hline 井冈電素 & 78 & -11 & 56 & 27 \\
\hline 甲基托布津 & 79 & 54 & 69 & 83 \\
\hline
\end{tabular}

\section{References}

[1] Liu, X. H.; Pan, L.; Weng, J. Q.; Tan, C. X.; Li, Y.H.; Wang, B. L.; Li, Z. M. Mol. Diversity 2012, 16, 251

[2] (a) Tan, C. X.; Shi, Y. X.; Weng, J. Q.; Liu, X. H.; Li, B. J.; Zhao, W. G. Lett. Drug Des. Discovery 2012, 9, 431.

(b) Liu, X. H.; Tan, C. X.; Weng, J. Q. Phosphorus, Sulfur Silicon Relat. Elem. 2011, 186, 558.

[3] Yin, K.; Jiang, L. H.; Zhou, H. X.; Huang, Y.; Xiang, J. N. Chin. J. Org. Chem. 2008, 28, 1016 (in Chinese)
(尹凯，蒋历辉，周后相，黄勇，向建南，有机化学，2008，22,71.)

[4] Kumar, D.; Narayanam, M. K.; Chang, K. H.; Shah, K. Chem. Biol. Drug Des. 2011, 77, 182-188.

[5] Al-Omar, M. A.; Al-Abdullah, E. S.; Shehata, I. A.; Habib, E. E.; Ibrahim, T. M.; El-Emam, A. A. Molecules 2010, 15, 2526.

[6] Kalhor, M.; Mobinikhaledi, A.; Dadras, A.; Tohidpour, M. J. Heterocycl. Chem. 2011, 48, 1366.

[7] Deng, X. Q.; Xiao, C. R.; Wei, C. X.; Quan, Z. S. Chin. J. Org. Chem. 2011, 31, 2082 (in Chinese)

(邓先清，肖春瑞，魏成喜，全哲山，有机化学，2011，31，2082.)

[8] (a) Li, S.; Cui, C.; Wang, M. Y.; Yu, S. J.; Shi, Y. X.; Zhang, X.; 
Li, Z. M.; Zhao, W. G.; Li, B. J. J. Fluorine Chem. 2012, 137, 108. (b) Zhou, J. K.; Song, B. A.; Xue, W.; Jin, L. H.; Gan, Z. Y. Chin. J. Org. Chem. 2011, 31, 865 (in Chinese).

(周进康, 宋宝安, 薛伟, 金林红, 干正洋, 有机化学, 2011, 31, 865.)

[9] Li, Q. Z.; Song, B. A.; Cai, X. J.; Zheng, Y. G.; Guo, Q. Q. Chin. J. Org. Chem. 2010, 30, 569 (in Chinese).

(李黔柱, 宋宝安, 蔡学建, 郑玉国, 郭晴晴, 有机化学, 2010, 30, 569.)

[10] (a) Liu, X. H.; Pan, L.; Ma, Y.; Weng, J. Q.; Tan, C. X.; Li, Y. H.;
Shi, Y. X.; Li, B. J.; Li, Z. M.; Zhang, Y. G. Chem. Biol. Drug Des. 2011, 78, 689.

(b) Liu, X. H.; Weng, J. Q.; Tan, C. X. Asian J. Chem. 2011, 23, 4064.

[11] He, F. Q.; Liu, X. H.; Wang, B. L.; Li, Z. M. Heteroat. Chem. 2008, 19, 21.

[12] Guo, Y. B.; Liu, H. B.; Xu, M. Chin. J. Org. Chem. 2012, 32, 413 (in Chinese).

(郭永彪, 刘海波, 许明, 有机化学, 2012, 32, 413.)

(Li, L.; Lu, Z.) 